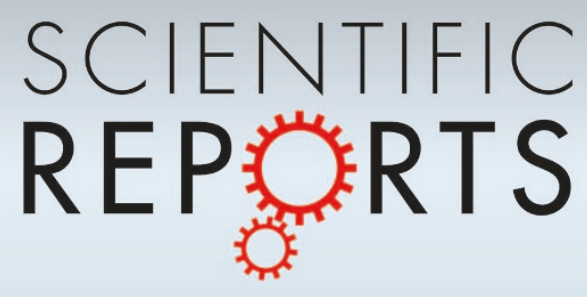

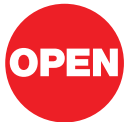

SUBJECT AREAS:

NANOWIRES

SYNTHESIS AND PROCESSING

ELECTRONIC PROPERTIES AND

MATERIALS

ELECTRONIC DEVICES

Received

24 September 2012

Accepted

15 January 2013

Published

15 February 2013

Correspondence and requests for materials should be addressed to K.K.N. (nanda@mrc. iisc.ernet.in)

\section{Uninterrupted and reusable source for the controlled growth of nanowires}

\author{
R. P. Sugavaneshwar \& Karuna Kar Nanda
}

Materials Research Centre, Indian Institute of Science, Bangalore-560012, INDIA.

Generally, the length of the oxide nanowires grown by vapor phase transport is limited by the degradation of the source materials. Furthermore, the source material is used once for the nanowires growth. By exploiting the $\mathrm{Si}-\mathrm{Zn}$ phase diagram, we have developed a simple methodology for the non-catalytic growth of ultralong $\mathrm{ZnO}$ nanowires in large area with controllable aspect ratio and branched structures. The insolubility of $\mathrm{Zn}$ in $\mathrm{Si}$ and the use of a Si cap on the $\mathrm{Zn}$ source to prevent local source oxidation of $\mathrm{Zn}$ (i. e. prevents the degradation of the source) are the keys to grow longer nanowires without limitations. It has been shown that the aspect ratio can be controlled by thermodynamically (temperature) and more importantly by kinetically (vapor flux). One of the interesting findings is that the same source material can be used for several depositions of oxide nanostructured materials.

T he vapor-liquid-solid (VLS) crystal growth mechanism has been widely used to guide the growth of a wide range of nanowires ${ }^{1}$, such as Group IV ( $\mathrm{Si}$ and Ge), III-V (GaAs, InAs, GaN and InP), and II-VI (CdS, CdSe, $\mathrm{ZnS}$ and $\mathrm{ZnSe}$ ) semiconductors and oxides $\left(\mathrm{ZnO}, \mathrm{Ga}_{2} \mathrm{O}_{3}, \mathrm{SnO}\right.$ and $\mathrm{SiO}_{2}$ ) and the heterostructures $^{2-15}$. A common morphological feature of VLS-grown nanowires is that each nanowire is terminated at one end by a catalyst nanoparticle with a comparable diameter. However, catalyst at the tip precludes the nanowires from a variety of applications including field emission, lasing, photovoltaic cells, etc. Though it is possible to remove the catalyst after growth, it also etches the nanowires ${ }^{8}$. Therefore, the requirement demands to synthesize catalyst free nanowires. Non-catalytic growth of several nanowires ( $\mathrm{Si}, \mathrm{Ge}, \mathrm{GaN}$, $\left.\mathrm{ZnO}, \mathrm{Ga}_{2} \mathrm{O}_{3}, \mathrm{CdSe}, \mathrm{B}\right)$ and their heterostructures have been possible ${ }^{16-26}$. Nanowires produced by noncatalytic growth mechanism seem to best suit the nanowire technology development as there is no involvement of catalytic nanoparticles. It is believed that non-catalytic growth proceeds in two steps ${ }^{26}$ : (1) nucleation of seed nanoparticles controlled by thermodynamic size limit and (2) subsequent one-dimensional anisotropic growth without catalyst.

The growth of the nanowires is a process governed by a competition between kinetics and thermodynamics. Theoretical descriptions on the catalytic as well as non-catalytic growth of nanowires are available in the literature ${ }^{26-29}$. The diameter of nanowires is mainly controlled by the size of the catalyst ${ }^{27-29}$, while the diameter of nanowires obtained by non-catalytic growth is governed by thermodynamic limit ${ }^{26}$. However, the length and hence, the aspect ratio of the nanowires, is expected to increase with growth duration. But the quest to improve aspect ratio is limited by various experimental shortcomings such as catalytic poisoning ${ }^{30}$, degradation of the precursors and growth in all possible directions ${ }^{31}$. Some efforts have been channelized in overcoming the above limitation such as by mixing $\mathrm{NaCl}$ with $\mathrm{Zn}$ to prevent degradation of precursors ${ }^{32}$.

Here, we report the non-catalytic synthesis of $\mathrm{ZnO}$ nanowires on soda lime glass (SLG) substrates and shown that the diameter of the nanowires is not only controlled by thermodynamically but also kinetically. It is shown that smaller diameter of the nanowires as compared to that set by the thermodynamic limit can be obtained. We have employed a technique wherein the source is protected from degradation ensuring the continuous supply of vapor. This is achieved by placing Si cap on $\mathrm{Zn}$ source. We have investigated the influence of temperature, growth duration and more importantly, the vapor flux. The results obtained are beyond the theoretical descriptions available in the literature. We have achieved ultralong $\mathrm{ZnO}$ nanowires $(>300 \mu \mathrm{m})$ with huge aspect ratio $\left(>10^{4}\right)$ and branched structures by controlling the growth both by thermodynamically and kinetically. Our results suggest the possibility of growing $\mathrm{ZnO}$ nanowires continuously without any apparent length limitation. Understanding the thermodynamic and kinetic controlled noncatalytic growth of nanowires is a great asset for the realization of longer and dense arrays of vertical nanowire for various applications. Furthermore, it is shown that the same source material can be used for several depositions of oxide nanowires. 


\section{Results}

Fig. 1a-e shows the $\mathrm{ZnO}$ nanowires obtained by heating $\mathrm{Zn}$ powders with Si cap (supplementary fig. 1) at $650^{\circ} \mathrm{C}$ for $5 \mathrm{~min} ., 1,2,5,10$ and $50 \mathrm{~h}$ on SLG placed at $645^{\circ} \mathrm{C}$. Optimum results have been obtained at a substrate temperature $T_{\text {sub }}$ of $645^{\circ} \mathrm{C}$ (distance between the source and substrate is $5 \mathrm{~mm}$ ). The length as a function of growth duration is shown in Fig. 1g. The length of the $\mathrm{ZnO}$ nanowires is found to increase almost linearly with time from $1 \mu \mathrm{m}$ in $5 \mathrm{~min}, 12 \mu \mathrm{m}$ in $1 \mathrm{~h}$, $20 \mu \mathrm{m}$ in $2 \mathrm{~h}, 112 \mu \mathrm{m}$ in $10 \mathrm{~h}$ and $300 \mu \mathrm{m}$ in $50 \mathrm{~h}$. The increase in length with increasing in time in the case $\mathrm{Zn}$ source with $\mathrm{Si}$ cap is attributed to continuous supply of $\mathrm{Zn}$ vapors throughout the experiment. Since there is no catalyst used, the growth of nanowires is attributed to vapor-solid (VS) or self-catalytic mechanism. The morphology was fully consistent with the growth habit of the $\mathrm{ZnO}$ ideal crystal model $^{33}$ in that the relative crystal growth velocity should follow the sequences: $V(0001)>V(01 \overline{1} 0)>V(000 \overline{1})$. The surface energy of (0001) surface is $2.0 \mathrm{~J} / \mathrm{m}^{2}$, while that of $(01 \overline{1} 0)$ surface is $1.16 \mathrm{~J} / \mathrm{m}^{2}{ }^{34}$. Branched structures are obtained by this method are shown in Fig. 1h \& i. The growth of branched structures was performed by keeping $\mathrm{ZnO}$ nanowires deposited on SLG at a distance of $5 \mathrm{~mm}$ from the source.

The nanowires can not only be grown on SLG but also on other substrates such as multiwalled carbon nanotubes (MWCNTs) bundles, indium tin oxide (ITO), and Si. Fig. $1 \mathbf{j}-\mathbf{l}$ shows $\mathrm{ZnO}$ nanowires

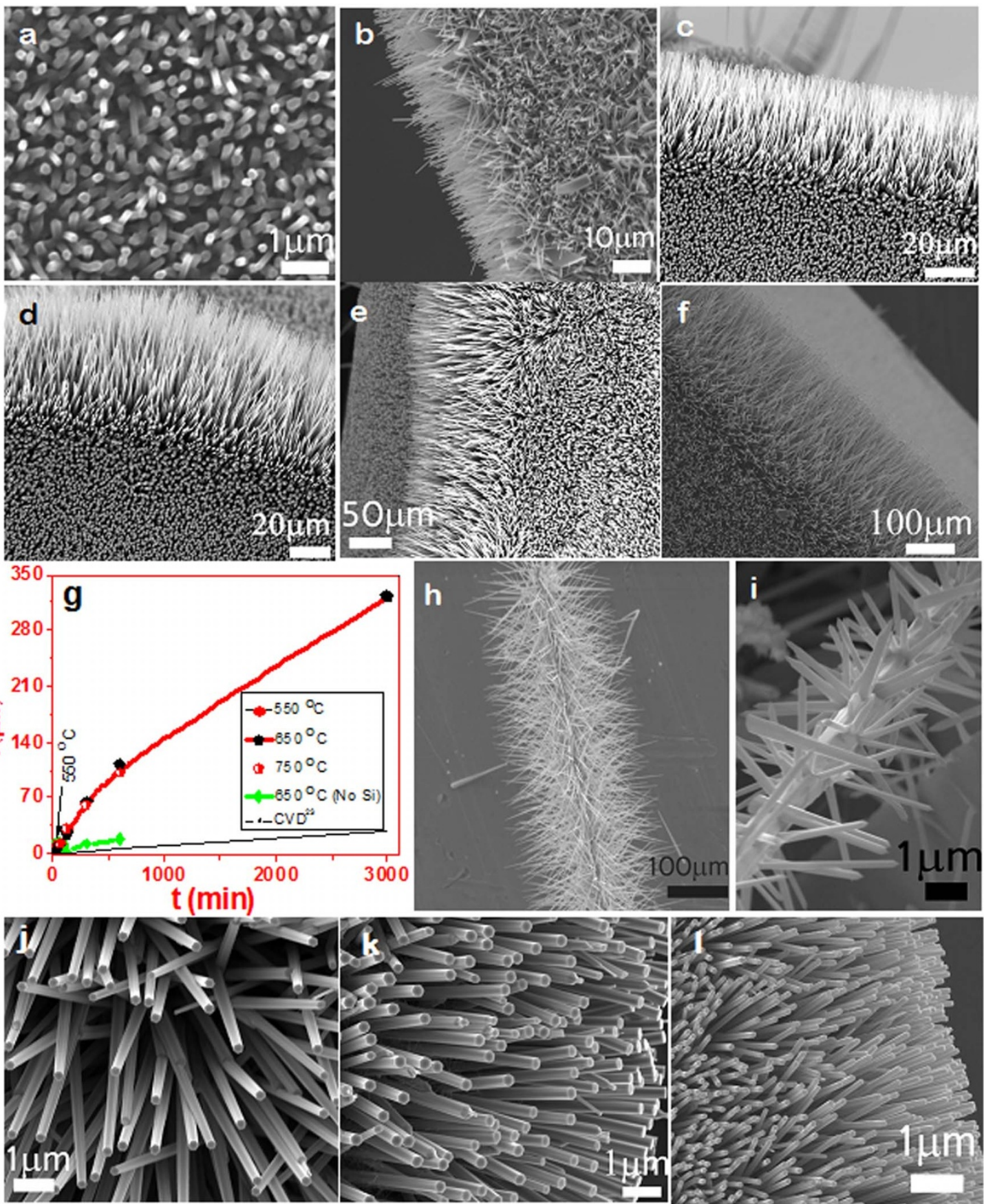

Figure $1 \mid \mathrm{ZnO}$ nanowires on SLG synthesized at $650^{\circ} \mathrm{C}$ for (a) $5 \mathrm{~min}$, (b) 1 , (c) 2, (d) 5 , (e) 10 (f) $50 \mathrm{~h}$, and (g) length as a function of growth duration for different temperatures. The dashed lines in $(\mathrm{g})$ are guide to the eye. Length of nanowires as obtained by chemical vapour deposition (CVD) is also plotted for comparison. ( $\mathrm{h} \& \mathrm{i}$ ) branched $\mathrm{ZnO}$ nanostructures. Regrowth of $\mathrm{ZnO}$ nanowires on already grown $\mathrm{ZnO}$ nanowires (grown at $650^{\circ} \mathrm{C}$ for $10 \mathrm{~h}$ ) on a SLG substrate yield branched structures. ZnO nanowires grown on (j) MWCNT, (k) Si, and (l) ITO. 
grown on MWCNT bundles, ITO and Si. The MWCNT growth was achieved by dispersing them on a SLG. The morphology obtained is uniform and similar to that obtained on SLG.

We have also investigated the growth of $\mathrm{ZnO}$ without the Si cap. The length of the nanowires (supplementary fig. 2) is around $15 \mu \mathrm{m}$ for $10 \mathrm{~h}$ deposition which is much smaller as compared to the length obtained with Si cap (length is $112 \mu \mathrm{m}$ ) but comparable with that obtained by using only zinc sources as reported in Ref. 24,25 where the length of the nanowires ranges from $2 \mu \mathrm{m}$ to maximum of $15 \mu \mathrm{m}$. This clearly suggests that the length cannot be increased further without the protection of the $\mathrm{Zn}$ source. It may also be noted from Fig. 1g that growth rate is much higher (at least ten times) as compared to the non-catalytic growth of nanowires by chemical vapor deposition $(\mathrm{CVD})^{21-23}$, and physical vapor transport technique without $\mathrm{Si} \mathrm{cap}^{24,25}$. We also observed that the growth rate increases with the rate of gas flow (supplementary fig. 3). The growth rate was increased from 12 to $20 \mu \mathrm{m} / \mathrm{h}$ when the gas flow rate was doubled.

Fig. 2a-c shows SEM images of $\mathrm{ZnO}$ nanowires obtained by heating $\mathrm{Zn}$ pellet at 550,650 and $750^{\circ} \mathrm{C}$ for $1 \mathrm{~h}$. The diameter of the $\mathrm{ZnO}$ nanowires reduced from $120 \mathrm{~nm}$ at $550^{\circ} \mathrm{C}$ to $46 \mathrm{~nm}$ at $750^{\circ} \mathrm{C}$ which is confirmed from the TEM images of the $\mathrm{ZnO}$ nanowires as shown in the insets. High resolution TEM (HRTEM) images taken from the side of the nanowires are shown in Fig. 2d-f which along with $\mathrm{x}$-ray diffraction data (supplementary figs $4 \& 5$ ) reveal the nanowire long axes are parallel to the [0002] direction. It may be noted that the density of nanowires increases and the diameter decreases as the temperature is increased, which is attributed to the increase in supersaturation of $\mathrm{Zn}$ vapours with increasing temperature. The minimum radius $R^{*}$ (the critical radius) can be predicted as it is given by $^{35,36}$

$$
R^{*}=\frac{\left(3.3 \sigma_{1}+\sigma_{12}\right) v}{k T_{s u b} \ln \left(p / p_{\infty}\right)}
$$

Here, $\sigma_{1}$ is the cluster surface energy $(\mathrm{ZnO}), \sigma_{12}$ is the cluster-substrate interface energy $(\mathrm{ZnO}-\mathrm{C}), v$ is the atomic volume, $k$ is Boltzmann's constant, $T_{\text {sub }}$ is the absolute temperature of the substrate, $p$ is the pressure of the precursor at $T_{s u b}$ and $p_{\infty}$ is the equilibrium vapor pressure at $T_{\text {source }}$ (the set/source temperature). Both $T_{\text {source }}$ and $T_{\text {sub }}$ at a distance of $5 \mathrm{~mm}$ were measured and provided in supplementary Table 1 . Based on the above equation and assuming that the $\mathrm{Zn}$ vapor condenses to form $\mathrm{ZnO}$ nanowires, the diameters at different temperatures were calculated and compared with the experimental results as shown in Fig. $2 \mathrm{~g}$. The theoretical results supported the decrease of diameter with increasing temperature but there is a quantitative difference. It may be noted that the diameter of nanowires increases with growth duration but very weakly as compared to the length. This corroborates the difference in the diameter and critical diameter as presented in Fig. $\mathbf{2 g}$.

According to Lifshitz-Slyozov-Wagner (LSW) theory ${ }^{37,38}$, the average diameter of the particles follows the relation $d^{3}=d_{0}^{3}+K t$ where $d$ is the average diameter at time $t$ and $d_{0}$ is the average initial diameter of the nanocrystals. The rate constant $K$ is given by $K=8 \gamma D V_{m}^{2} C_{\infty} / 9 R T$, where $D$ is the diffusion constant at temperature $T, V_{m}$ is the molar volume, $\gamma$ is the surface energy, and $C_{\infty}$ is the equilibrium concentration at a flat surface. For nanowire growth with negligible increase in the diameter, the length is expected to be linear with time. As the diameter is almost independent of time, LSW relation can clearly be rewritten as

$$
l=l+\alpha t
$$

where $l$ represents the length of nanowires and $\alpha$ is the proportionality constant that depends on the diameter of the nanowires. Smaller is the diameter, larger is the increase in the length. Our experimental results (Fig. 1g) are consistent with the theoretical descriptions. Interestingly, the length is almost independent of temperature and follows a sacred relation with growth duration as evident from Fig. 1g. As discussed later, the growth of nanowires occurs through diffusion of adatoms onto the sidewalls. It has been argued for surface diffusion on sidewalls of nanowires that the length of the nanowires should be inversely proportional to the diameter ${ }^{39}$,

$$
l=\frac{1}{D}+c
$$

where $c$ is constant. Accordingly, the length should increase with temperature as the diameter is decreasing. However, the increase in density along with the decrease in diameter with increase in the growth temperature results in the temperature independent length.

As the degree of supersaturation is increased, number of nuclei increases, subsequently leading to the reduction in the diameter of the nanowire. Saunders et $\mathrm{al}^{40}$ predicted that the degree of supersaturation of $\mathrm{Zn}$ first increases and then decreases as the temperature is increased during the formation of $\mathrm{ZnO}$ by vapour phase transport,
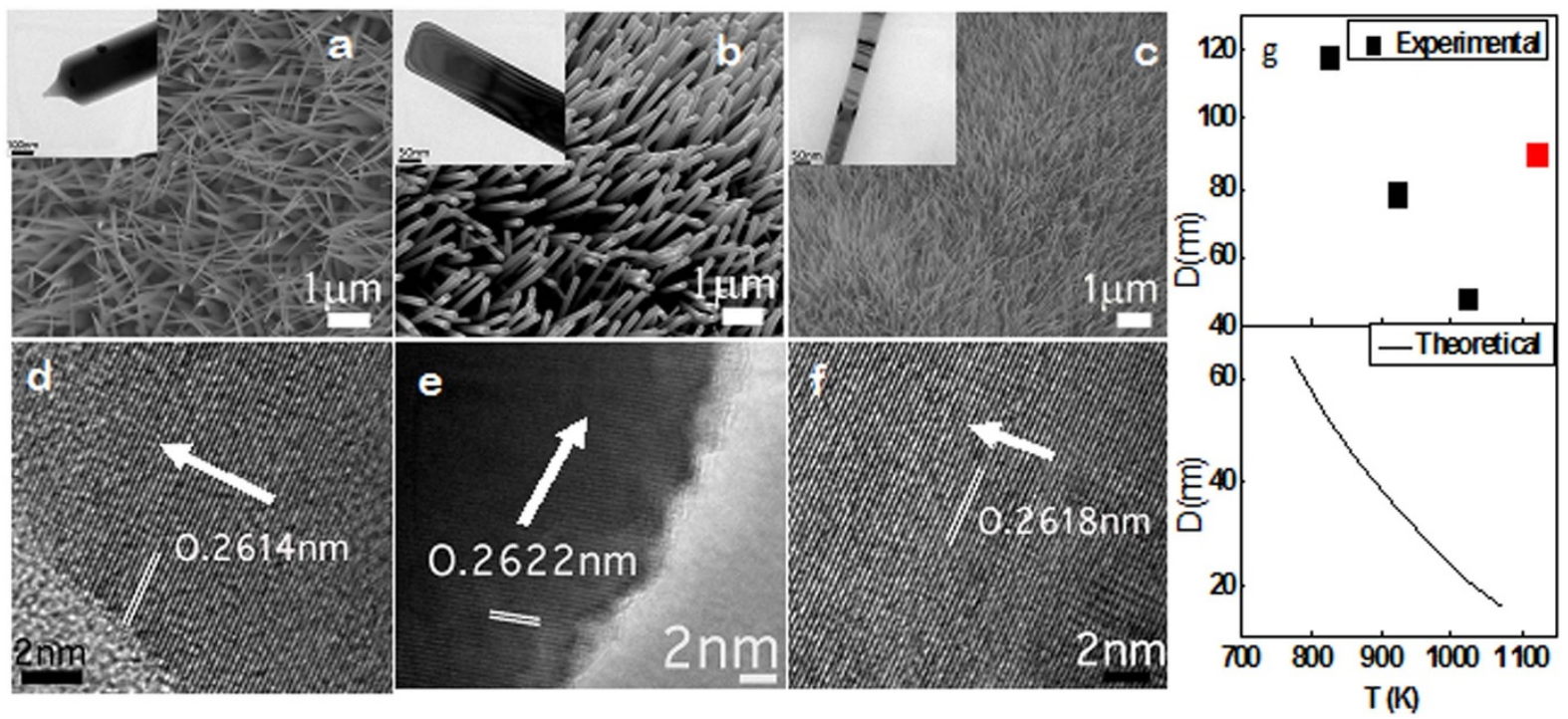

Figure $2 \mid(\mathrm{a}-\mathrm{c})$ SEM and (d-f) HRTEM images of ZnO nanowires on SLG by heating at 550,650 and $750^{\circ} \mathrm{C}$ for $1 \mathrm{~h}$. TEM images of selected nanowires are shown in the insets. (g) Diameter (experimental) and critical diameter (theoretical) as a function of temperature. The red square represents the diameter of nanowires grown on a quartz substrate. 

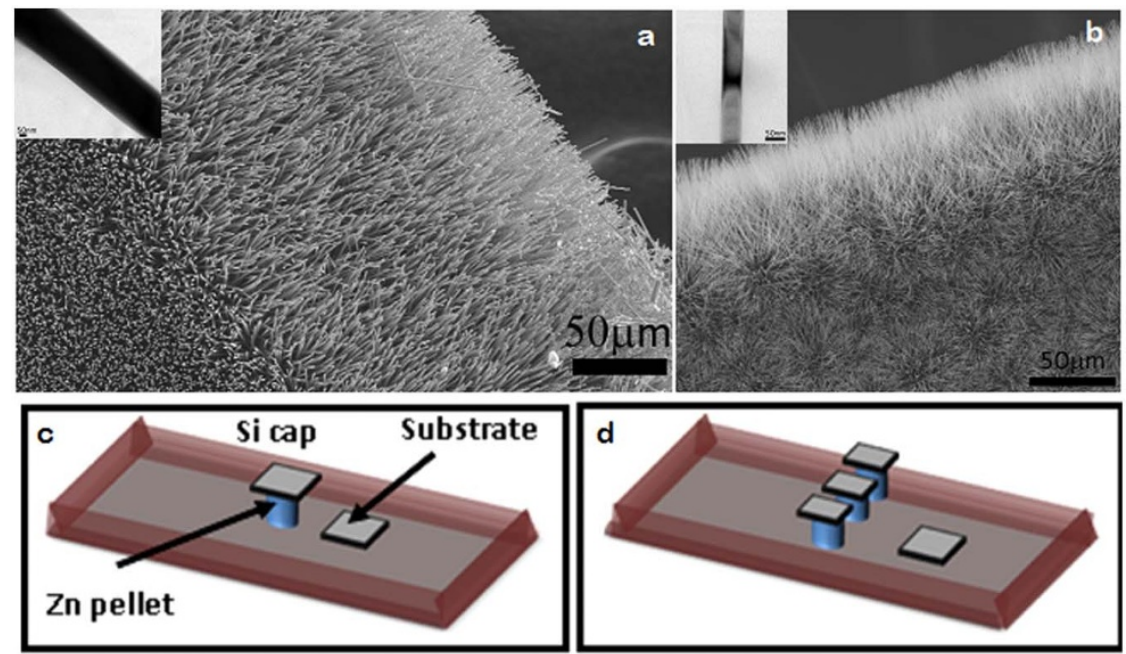

Figure 3 SEM images of ZnO nanowires on glass obtained by increasing the vapour flux (a) single source and (b) triple source with TEM images in the inset. Schematic of (c) single and (d) triple source configurations.

thereby implying that it would be impossible to reduce the diameter of the nanowires with increasing temperature beyond certain limit. We have grown $\mathrm{ZnO}$ nanowires (supplementary fig. 6) on a quartz substrate (as SLG cannot withstand the temperature) at $1123 \mathrm{~K}$ and the diameter is found to be $\sim 80 \mathrm{~nm}$ as indicated in Fig. $2 \mathrm{~g}$ by red square. This supports that the diameter of nanowires cannot be reduced beyond certain limit. Here we propose an alternate approach by which the diameter can be reduced further. We have shown that the diameter can be reduced by using more than one source to obtain more vapour flux without increasing the temperature.

SEM images shown in Fig. 3 are obtained by using one and three sources at $650^{\circ} \mathrm{C}$. This indicates that the vapour flux is three times in the later case. TEM images of selected nanorods are shown in the insets. It is interesting to note that the diameter decreases as the flux
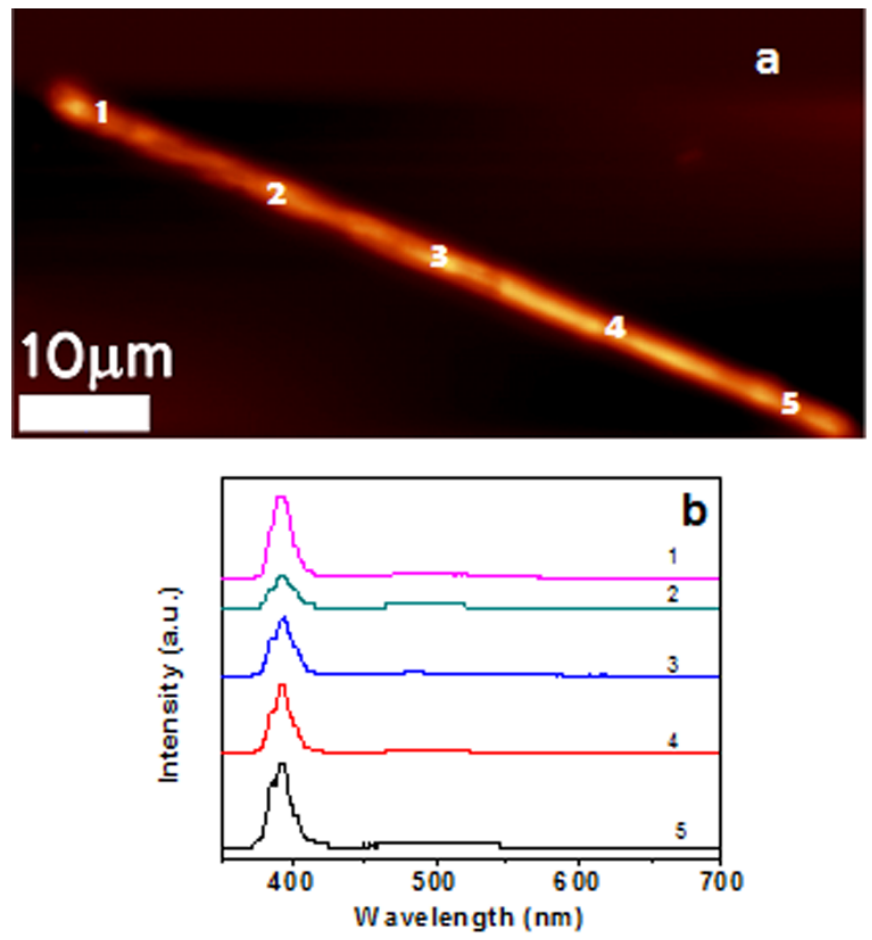

Figure $4 \mid$ (a) PL imaging $\left(\lambda_{\text {excitation }}=355 \mathrm{~nm}\right.$ and $\lambda_{\text {detection }}=380 \mathrm{~nm}$ ) (b) PL spectra at different positions of an isolated nanowire. is increased. The diameter of the $\mathrm{ZnO}$ nanorods is $\sim 120 \mathrm{~nm}$ when single source is used while diameter reduces to $\sim 40 \mathrm{~nm}$ when three sources is used as confirmed by TEM studies. Over all, aspect ratios greater than $10^{4}$ can easily be achieved by this technique (supplementary fig. 7).

We believe that $\mathrm{ZnO}$ particles nucleates first on the substrate ${ }^{24-26,35}$. After reaching the thermodynamic size limit, nanowire growth proceeds in the [0001] direction (supplementary fig. 8). Nanowire growth is primarily due to the adsorption of adatoms onto the sidewalls of nanowires and subsequent diffusion to the tips. This is consistent with the growth of long nanowires at long growth times. Branched structures are obtained when $\mathrm{ZnO}$ nanowires were grown on nanowires as shown in Fig. 1i. This also supports the adsorption of adatoms onto the sidewalls of nanowires and the formation of clusters followed by the growth of the nanowires.

There are two possible path ways for the growth of $\mathrm{ZnO}$ nanowires ${ }^{41}$. One is the formation of $\mathrm{Zn}$ nuclei followed by oxidation and growth, while the other one is the formation of $\mathrm{ZnO}$ nuclei followed by growth. As the oxidation of $\mathrm{Zn}$ is prevented, it is very likely that $\mathrm{Zn}$ vapors condense on $\mathrm{ZnO}$ in our case. However, as the source used in Ref. 24 is exposed to oxygen, the surface is expected to oxidize completely and $\mathrm{ZnO}$ vapor species are obtained. Variation of the degree
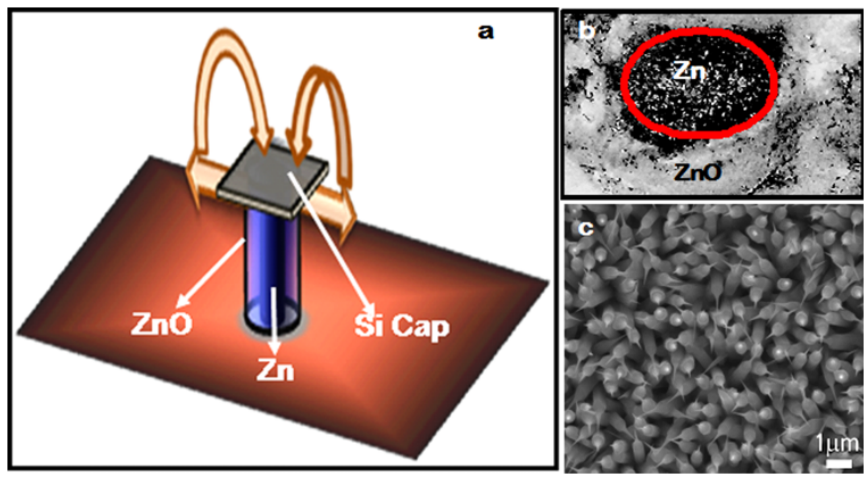

Figure $5 \mid$ (a) Schematic of the formation $\mathrm{ZnO}$ nanowires on Si cap placed over $\mathrm{Zn}$ powder. (b) Photograph of the pellet recovered after the completion of experiment. Dark region $(\mathrm{Zn})$ inside red circle indicates the region of the pellet was in contact with the Si substrate during the experiment. (c) SEM image of $\mathrm{ZnO}$ nanoneedles on Si placed on $\mathrm{Zn}$ pellet. Nanoneedles are obtained for a growth temperature of $650^{\circ} \mathrm{C}$ for $1 \mathrm{~h}$. 
of supersaturation with temperature is shown to be different for different pathways ${ }^{40}$. The different variation of diameter with temperature as discussed earlier can be due to the different degree of supersaturation. We would also like to point out that the vapors cannot be supplied continuously if the source is not protected from oxidation. The low growth rate reported in Ref. 24 is therefore lower as compared to ours. The source material cannot be changed as it may lead to the growth of branched structures without increasing the length of the nanowires.

The photoluminescence (PL) spectra of $\mathrm{ZnO}$ nanowires (supplementary fig. 9) exhibit strong peaks at $380 \mathrm{~nm}$ in all cases suggesting the formation of high quality nanorods. There is also a weak broad peak around $530 \mathrm{~nm}$ which is attributed to oxygen deficiencies ${ }^{40-42}$. It may be noted that the PL peak at $530 \mathrm{~nm}$ is significant for $\mathrm{ZnO}$ nanowires synthesized at low temperature with one source for $1 \mathrm{~h}$ as compared to other nanowires. Raman spectra (supplementary fig. 9) shows peaks at 333, 438, 480 and $560 \mathrm{~cm}^{-1}$ that match the position of the characteristic peaks of $\mathrm{ZnO}^{43}$. Fig. $4 \mathrm{a} \& \mathrm{~b}$ show the PL imaging and corresponding PL spectra taken at different positions along the length of the nanowire. The strong peak around $380 \mathrm{~nm}$ is almost uniform along the length indicating that nanowire is optically uniform.
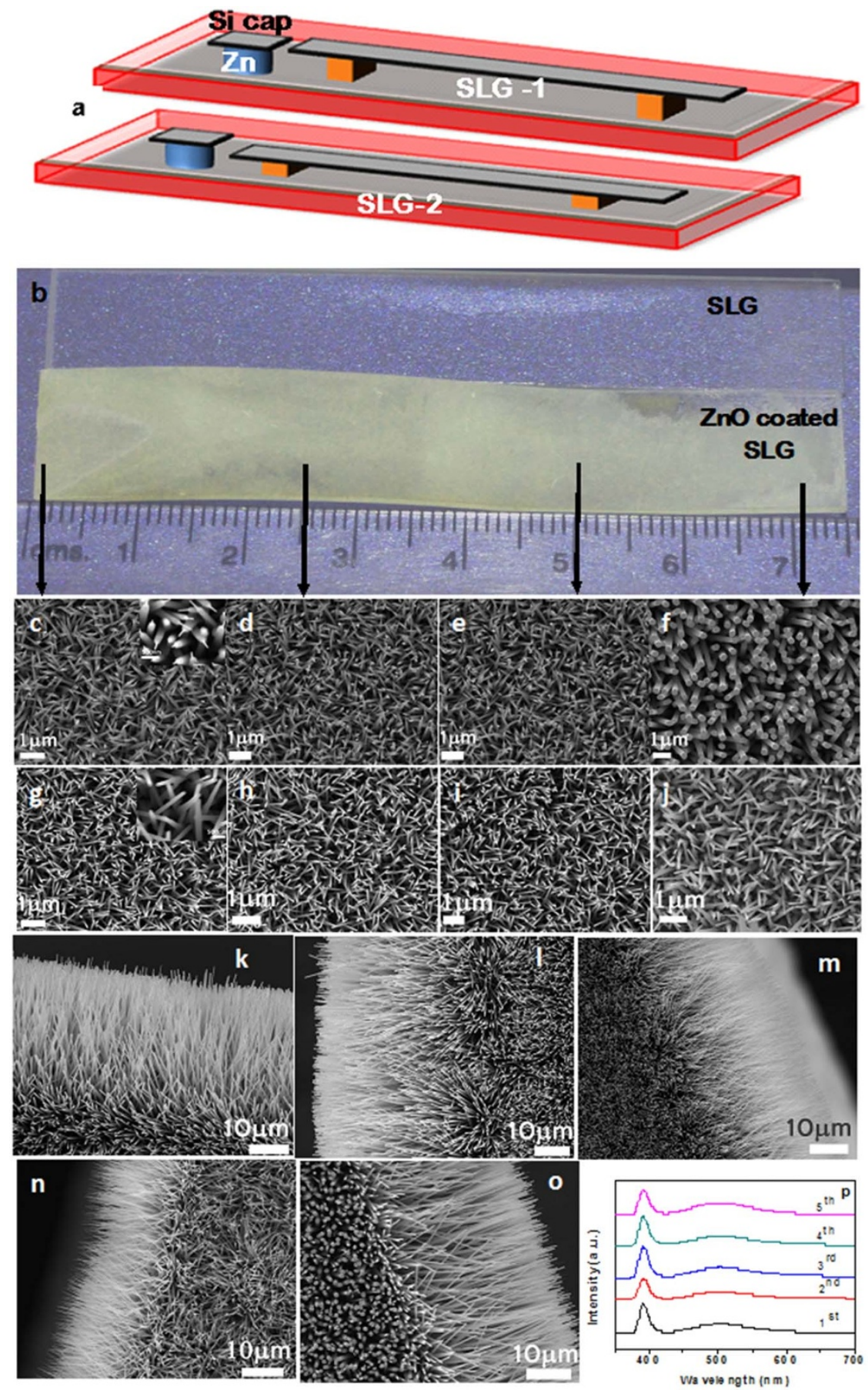

Figure $6 \mid$ (a) Schematic for the large area synthesis of ZnO nanowires/nanoneedles. (b) Optical photographs of a bare SLG and a SLG with ZnO nanoneedles. The area of the SLG is $\sim 75 \times 15 \mathrm{~mm}^{2}$. (c-f) SEM images of ZnO nanoneedles taken from different region of SLG-1. (g-j) SEM images of nanowires grown on SLG-2. High magnification images are shown in the insets of (c \& g). ZnO nanowires grown on SLG at $650^{\circ} \mathrm{C}$ for $2 \mathrm{~h}$. (k) $1^{\text {st }}$, (l) $2^{\text {nd }}$, (m) $3^{\text {rd }},(\mathrm{n}) 4^{\text {th }}$, and (o) $5^{\text {th }}$ subsequent deposition using the same source. (p) PL spectra of ZnO nanorods. 
The controlled growth of $\mathrm{ZnO}$ could be explained by using $\mathrm{Si}-\mathrm{Zn}$ phase diagram (Supplementary fig. 10) ${ }^{44}$. The maximum solid solubility of $\mathrm{Zn}$ in $\mathrm{Si}$ is 0.0015 at\%. When $\mathrm{Zn}$ pellet is heated while a part of it is in contact with Si cap as shown in Fig. 5a, the pellet gets oxidized except the surface in contact with Si. The optical photograph of the pellet recovered after the experiment is shown in Fig. 5b. Dark region inside red circle indicates the region of the pellet was in contact with the Si cap during the experiment and remains protected from oxidation. As the vapor pressure of $\mathrm{Zn}$ is much higher as compared to $\mathrm{ZnO}, \mathrm{Zn}$ vapors escape through the $\mathrm{Zn} / \mathrm{Si}$ interface and the higher pressure in that region prevent ingress of $\mathrm{O}_{2}$ and thus eliminates $\mathrm{Zn}$ oxidation in this region. The vapor is then transported to nearby regions and also onto the top of $\mathrm{Si}$ cap to form $\mathrm{ZnO}$ nanoneedles as shown in Fig. 5c. The solid solubility of $\mathrm{Zn}$ being small, $\mathrm{Zn}$ vapors do not react with $\mathrm{Si}$ and are not impeded from reaching the various substrates. Over all, the Si cap protects $\mathrm{Zn}$ in contact with it from oxidation thereby, acting as oxidation barrier, ensuring continuous vapor supply from $\mathrm{Zn}$ pellet leading to the controlled growth of $\mathrm{ZnO}$. This is in contrast to the case of a "bare" $\mathrm{Zn}$ pellet ${ }^{24,25}$ whose surfaces oxidize and $\mathrm{Zn}$ supply consequently reduces over time. It may also be noted that the diameter is found to be reduced as the temperature is increased which is in contrast with that reported in Ref. 24 where the increase of diameter with increasing temperature has been reported.

One of the challenges is the large area deposition. Fig. 6 shows the summary of the large area $\left(75 \times 15 \mathrm{~mm}^{2}\right)$ deposition of $\mathrm{ZnO}$ nanowires on SLG. A SLG substrate (SLG-1) was placed on a support so that the height can be equal to that of Si cap placed on $\mathrm{Zn}$ pellet as schematically shown in the Fig. 6a. Another SLG substrate (SLG-2) is placed at a different height in a separate experiment. The optical photograph of $\mathrm{ZnO}$ grown at $650^{\circ} \mathrm{C}$ for $2 \mathrm{~h}$ on SLG-1 is shown in Fig. $6 \mathrm{~b}$ which clearly reveals a uniform white deposition over length and breadth of the SLG substrate. SEM images of $\mathrm{ZnO}$ nanostructures taken from different location are shown in Fig. 6c-e which reveals needles-like morphology. It is interesting to note that the morphology and the dimensions of $\mathrm{ZnO}$ nanoneedles are identical over a large area of $60 \times 15 \mathrm{~mm}^{2}$ and identical to that grown on $\mathrm{Si}$ cap. The morphology beyond $60 \mathrm{~mm}$ is different as shown in Fig. 6 f. Similarly, nanorods-like morphology is obtained for $\mathrm{ZnO}$ grown on SLG-2 as shown in Fig. 6g-i. The morphology is identical over a large area of $60 \times 15 \mathrm{~mm}^{2}$ and is different beyond $60 \mathrm{~mm}$ (Fig. $6 \mathbf{j}$ ). These results indicate that the methodology adopted here is of a great promise for scale-up synthesis of $\mathrm{ZnO}$ nanowires. Over all, the diameter is increasing and the morphologies are different beyond $60 \mathrm{~mm}$ which may be due to difference in growth temperature.

Generally, the source material is used once for the oxide nanowires growth by vapour phase transport method. As the Si cap protects the source from the oxidation, we have investigated the reuse of source materials to grow nanowires. The substrates were replaced after each deposition without disturbing the source and Si cap. Fig. 6k-o show $\mathrm{ZnO}$ nanowires which reveal that the morphology of the $\mathrm{ZnO}$ nanowires is almost identical at least upto five subsequent depositions. The PL spectra as shown in Fig. 6p also reveal almost the same optical quality nanorods. The strong peak at $380 \mathrm{~nm}$ peak corresponds to the band-to-band transition and a weak broad peak around $\sim 515 \mathrm{~nm}$ is due to the oxygen vacancy. Over all, the same source material can be used for several depositions of oxide nanostructured materials.

\section{Discussion}

We have developed a simple methodology to grow ultralong nanowires and branched structures with controllable aspect ratio without limitations in a large area. We demonstrate the significance and importance of local oxidation barrier on source for the growth of ultralong nanowires, and more importantly for the first time, controlling the diameter of the nanowire kinetically without catalyst particle or seed layer. The insolubility of $\mathrm{Zn}$ in $\mathrm{Si}$ and the use of a $\mathrm{Si}$ cap on the $\mathrm{Zn}$ source to prevent oxidation is the key to grow ultralong nanowires. It has been shown that the aspect ratio can be controlled by thermodynamically (temperature) and kinetically (vapor flux). Over all, the length can be increased by increasing the deposition time and the diameter can be reduced by increasing the temperature and/or vapor flux. It is shown that smaller diameter of the nanowires as compared to that set by the thermodynamic limit can be obtained. The same source material can be used for several depositions of oxide nanostructured materials and suitable combination of materials can help to grow other oxide/sulfide nanostructures and oxide-sulfide branched structures in a controlled way.

For practical applications, selective growth of $\mathrm{ZnO}$ nanowires on pre-patterned substrates is required ${ }^{45-51}$. Our preliminary results indicate the possibility of nanowires growth on pre-patterned substrates (supplementary fig. 11). Furthermore, the methodology described here along with that reported in Refs. 52 \& 53 can be adopted to grow nanowires on a diverse range of pre-patterned substrates with or without catalysts.

\section{Methods}

A Si cap ${ }^{54}$ is placed over $\mathrm{Zn}$ pellets (Supplementary Fig. 1) which prevents oxidation of $\mathrm{Zn}$ (which served as local oxidation barrier for $\mathrm{Zn}$ ) and helps to grow longer nanowires without limitations. $\mathrm{Zn}$ pellets are made from $10 \mathrm{mg}$ of $\mathrm{Zn}$ powders (S.D fine $99.95 \%$ ) and along with Si cap is placed at the centre of the quartz boat. The whole assembly is then kept inside the alumina tube on a single zone furnace

(Supplementary Fig. 1). The growth substrates are placed at different distance of $5 \mathrm{~mm}$ from the source ( $\mathrm{Zn}$ pellet with Si cap on top) and heated to various temperatures for different time duration under argon gas (99.99\%) flow of 110-220 sccm (standards cubic centimetre per minute). The flow is controlled by a mass flow controller (MFC). The experiments are performed at atmospheric pressure and the outlet is connected to the exhaust. After the experiment, the product is analysed by Scanning electron microscopy (SEM) using SEM Quanta 200 and transmission electron microscopy (TEM) analysis using TEM technai G-20. Raman spectra were recorded using WITec alpha 200 and the photoluminescence (PL) spectra were recorded using a Jobin Yvon system with He-Cd laser with 325 excitation wavelength and WITec alpha 200 with Nd-YAG laser with 355 excitation wavelength.

1. Wagner, R. S. \& Ellis, W. C. Vapour-liquid-solid mechanism of single crystal growth. Appl. Phys. Lett. 4, 89-90 (1964).

2. Morales, A. M. \& Lieber, C. M. A laser ablation method for the synthesis of crystalline semiconductor nanowires. Science 279, 208-211 (1998).

3. Yang, P. et al. Controlled growth of $\mathrm{ZnO}$ nanowires and their optical properties. Adv. Funct. Mater. 12, 323-331 (2002).

4. Jensen, L. E. et al. Role of surface diffusion in chemical beam epitaxy of InAs nanowires. Nano. Lett. 4, 1961-1964 (2004).

5. Park, W. II. et al. Controlled synthesis of millimeter-long silicon nanowires with uniform electronic properties. Nano. Lett. 8, 3004-3009 (2008).

6. Wang, Y. et al. Catalytic growth of large-scale single-crystal CdS nanowires by physical evaporation and their photoluminescence. Chem. Mater. 14, 1773-1777 (2002).

7. Liang, C. H. et al. Catalytic synthesis and photoluminescence of $\beta-\mathrm{Ga}_{2} \mathrm{O}_{3}$ nanowires. Appl. Phys. Lett. 78, 3202-3204 (2001).

8. Woodruff, J. H. et al. Vertically oriented germanium nanowires grown from gold colloids on silicon substrates and subsequent gold removal. Nano. Lett. 7, 1637-1642 (2007).

9. Hersee, S. D., Sun, X. \& Wang, X. The controlled growth of GaN nanowires. Nano. Lett. 6, 1808-1811 (2006).

10. Bakkers, E. P. A. M. et al. Epitaxial growth of InP nanowires on germanium. Nat. Mater. 3, 769-773 (2004)

11. Duan, X. \& Lieber, C. M. General synthesis of compound semiconductor nanowires. Adv. Mater. 12, 298-302 (2000).

12. Ma, C. et al. Single-crystal CdSe nanosaws. J. Am. Chem. Soc. 126, 708-709 (2003).

13. Gudiksen, M. S. et al. Growth of nanowire superlattice structures for nanoscale photonics and electronics. Nature 415, 617-620 (2002).

14. Wu, Y., Fan, R. \& Yang, P. Block-by-block growth of single-crystalline Si/SiGe superlattice nanowires. Nano. Lett. 2, 83-86 (2002).

15. Zhang, Y., Tang, Y., Lee, K. \& Ouyang, M. Catalytic and catalyst-free synthesis of CdSe nanostructures with single-source molecular precursor and related device application. Nano. Lett. 9, 437-441 (2008).

16. Greene, L. E. et al. General route to vertical $\mathrm{ZnO}$ nanowire arrays using textured ZnO seeds. Nano. Lett. 5, 1231-1236 (2005).

17. Kim, B. S. et al. Catalyst-free growth of single-crystal silicon and germanium nanowires. Nano. Lett. 9, 864-869 (2009). 
18. Vanithakumari, S. C. \& Nanda, K. K. A one-step method for the growth of $\mathrm{Ga}_{2} \mathrm{O}_{3}$ nanorod-based white-light-emitting phosphors. Adv. Mater. 21, 3581-3584 (2009).

19. Wang, L. et al. The plasmonic resonant absorption in GaN double-channel high electron mobility transistors. Appl. Phys. Lett. 99, 063502 (2011)

20. Cao, L. M. et al. Template-catalyst-free growth of highly ordered boron nanowire arrays. Appl. Phys. Lett. 80, 4226-4228 (2002).

21. Park, W. I., Kim, D. H., Jung, S. W. \& Yi, G. C. Metalorganic vapor-phase epitaxial growth of vertically well-aligned $\mathrm{ZnO}$ nanorods. Appl. Phys. Lett. 80, 4232-4234 (2002).

22. Park, W. I., Yi, G. C., Kim, M. \& Pennycook, S. J. ZnO nanoneedles grown vertically on Si substrates by non-catalytic vapor-phase epitaxy. Adv. Mater. 14, 1841-1843 (2002)

23. Park, W. I., Yi, G. C., Kim, M. Y. \& Pennycook, S. J. Quantum confinement observed in $\mathrm{ZnO} / \mathrm{ZnMgO}$ nanorod heterostructures. Adv. Mater. 15, 526-529 (2003).

24. Lyu, S. C. et al. Low-temperature growth of $\mathrm{ZnO}$ nanowire array by a simple physical vapor-deposition method. Chem. Mater. 15, 3294-3299 (2003).

25. Umar, A., Suh, E. K. \& Hahn, Y. B. Non-catalytic growth of high aspect-ratio ZnO nanowires by thermal evaporation. Solid State Commun. 139, 447-451 (2006).

26. Kwon, S. J. Theoretical analysis of non-catalytic growth of nanorods on a substrate. J. Phys. Chem. B 110, 3876-3882 (2006).

27. Tan, T. Y., Li, N. \& Gosele, U. Is there a thermodynamic size limit of nanowires grown by the vapor-liquid-solid process? Appl. Phys. Lett. 83, 1199-1201 (2003).

28. Wang, C. X., Wang, B., Yang, Yu-H. \& Yang, G.-W. Thermodynamic and kinetic size limit of nanowire growth. J. Phys. Chem. B 109, 9966-9969 (2005).

29. Liu, Q. X., Wang, C. X., Xu, N. S. \& Yang, G. W. Nanowire formation during catalyst assisted chemical vapor deposition. Phys. Rev. B 72, 085417 (2005).

30. Wang, Z. L. Zinc oxide nanostructures: growth, properties and applications. J. Phys.: Condens. Matter 16, R829-R858 (2004).

31. Zhuo, R. F. et al. Multistep synthesis, growth mechanism, optical, and microwave absorption properties of $\mathrm{ZnO}$ dendritic nanostructures. J. Phys. Chem. C 112, 11767-11775 (2008).

32. Wang, W. Z. et al. Aligned ultralong $\mathrm{ZnO}$ nanobelts and their enhanced field emission. Adv. Mater. 18, 3275-3278 (2006).

33. Li, W. J., Shi, E. W., Zhong, W. Z. \& Yin, Z. W. Growth mechanism and growth habit of oxide crystals. J. Cryst. Growth 203, 186-196 (1999).

34. Wander, A. et al. Stability of polar oxide surfaces. Phys. Rev. Lett. 86, 3811-3814 (2001).

35. Ok, J. G. et al. Electrically addressable hybrid architectures of Zinc Oxide nanowires grown on aligned carbon nanotubes. Adv. Funct. Mater. 20, 2470-2480 (2010).

36. Balluffi, R. W., Allen, S. M. \& Carter, W. C. Kinetics of materials. (John Wiley \& Sons, Inc, Hoboken, NJ, 2005).

37. Lifshitz, I. M. \& Slyozov, V. V. The kinetics of precipitation from supersaturated solid solutions. J. Phys. Chem. Solids 19, 35-50 (1961).

38. Wagner, C. Theorie der Alterung von Niederschlägen durch Umlösen (OstwaldReifung). Elektrochem, 65, 581-591 (1961).

39. Kim, D. S., Gösele, U. \& Zacharias, M. Surface-diffusion induced growth of $\mathrm{ZnO}$ nanowires. J. Cryst. Growth 311, 3216-3219 (2009).

40. Saunders, R. B., McGlynn, E. \& Henry, M. O. Theoretical analysis of nucleation and growth of $\mathrm{ZnO}$ nanostructures in vapor phase transport growth. Cryst. Growth Des. 11, 4581-4587 (2011).

41. Im, S., Jin, B. J. \& Yi, S. Ultraviolet emission and microstructural evolution in pulsed-laser-deposited ZnO films. J. Appl. Phys. 87, 4558-4561 (2000).

42. Djurišić, A. B. \& Leung, Y. H. Optical properties of ZnO nanostructures. Small 2, 944-961 (2006).
43. Ozgur, U. et al. A comprehensive review of $\mathrm{ZnO}$ materials and devices. J. Appl. Phys. 98, 041301 (2005).

44. Olesinski, R. \& Abbaschian, G. The Si-Zn (Silicon-Zinc) system. J. Phase Equilibria 6, 545-548 (1985).

45. Ahsanulhaq, Q., Kim, J. H. \& Hahn, Y.-B. Etch-free selective area growth of wellaligned $\mathrm{ZnO}$ nanorod arrays by economical polymer mask for large-area solar cell applications. Solar Energy Materials and Solar Cells 98, 476-481 (2012).

46. Ahsanulhaq, Q., Kim, J. H., Lee, J. S. \& Hahn, Y. B. Electrical and gas sensing properties of $\mathrm{ZnO}$ nanorod arrays directly grown on a four-probe electrode system. Electrochemistry Communications 12, 475-478 (2010).

47. Qurashi, A., Kim, J. H. \& Hahn, Y.-B. Direct fabrication of $\mathrm{ZnO}$ nanorods array on-chip system in solution and their electrical properties. Electrochemistry Communications 18, 88-91 (2012).

48. Hahn, Y.-B. Zinc oxide nanostructures and their applications. Korean J. Chem. Eng. 28, 1797-1813 (2012).

49. Ahsanulhaq, Q., Kim, J.-H. \& Hahn, Y.-B. Controlled selective growth of $\mathrm{ZnO}$ nanorod arrays and their field emission properties. Nanotechnology 18 (48), 485307 (2007).

50. Park, Y. K., Choi, H. S., Kim, J.-H., Kim, J.-H. \& Hahn, Y.-B. High performance field-effect transistors fabricated with laterally grown $\mathrm{ZnO}$ nanorods in solution. Nanotechnology 22, 185310 (2011).

51. Ahsanulhaq, Q., Kim, J., Kim, J. \& Hahn, Y. B. Seedless Pattern Growth of QuasiAligned ZnO Nanorod Arrays on Cover Glass Substrates in Solution. Nanoscale Res. Lett. 5, 669-674 (2010).

52. Wang, X., Summers, C. J. \& Wang, Z. L. Large-scale hexagonal-patterned growth of aligned $\mathrm{ZnO}$ nanorods for nano-optoelectronics and nanosensor arrays. Nano Lett. 4, 423-426 (2004).

53. Byrne, D., McGlynn, E., Cullen, J. \& Henry, M. O. A catalyst-free and facile route to periodically ordered and c-axis aligned $\mathrm{ZnO}$ nanorod arrays on diverse substrates. Nanoscale 3, 1675-1682 (2011).

54. Yan, Y., Zhou, L., Han, Z. \& Zhang, Y. Growth analysis of hierarchical ZnO nanorod array with changed diameter from the aspect of supersaturation ratio. J. Phys. Chem.C 114, 3932-3936 (2010).

\section{Acknowledgments}

The authors acknowledge Department of Information Technology (DIT) through Nanotechnology programme for financial support. Authors also acknowledge Dr. S. Raghavan, Dr. Enda McGlynn and Dr. Abhishek Kumar Singh for their helpful suggestions.

\section{Author contributions}

R.P.S. performed experiments. Both R.P.S. and K.K.N. analyzed the data. K.K.N. wrote the paper.

\section{Additional information}

Supplementary information accompanies this paper at http://www.nature.com/ scientificreports

Competing financial interests: The authors declare no competing financial interests. License: This work is licensed under a Creative Commons Attribution-NonCommercial-NoDerivs 3.0 Unported License. To view a copy of this license, visit http://creativecommons.org/licenses/by-nc-nd/3.0/

How to cite this article: Sugavaneshwar, R.P. \& Nanda, K.K. Uninterrupted and reusable source for the controlled growth of nanowires. Sci. Rep. 3, 1172; DOI:10.1038/srep01172 (2013) 